\title{
A facilitação de uma comunidade de prática de professores de natação: um relato de experiência
}

\author{
Facilitating a community of practice for swimming teachers: an experience report
}

\author{
Leonardo Ristow \\ Centro Universitário de Brusque (Unifebe), Brusque, Brasil
}

\section{HISTÓRICO DO ARTIGO \\ Recebido: 21 dezembro 2020 \\ Revisado: 04 março 2021}

Aprovado: 23 março 2021

\section{PALAVRAS-CHAVE:}

Natação; Comunidade de prática; Desenvolvimento profissional.

\section{KEYWORDS:}

Swimming; Community of Practice; Professional Development.

\section{RESUMO}

INTRODUÇÃO: Comunidades de prática são grupos de profissionais que compartilham de um mesmo interesse. Um elemento essencial para conduzi-las é o facilitador. Este, tem a função de organizar os encontros, estimular a cooperação e auxiliar os membros na aprendizagem profissional.

OBJETIVO: Relatar a experiência vivenciada na facilitação de uma comunidade de prática de professores de natação.

MÉTODOS: Foi realizado um estudo descritivo na modalidade de relato de experiência do facilitador de uma comunidade de prática professores de natação. Participaram desta comunidade três professores, um que atuou como facilitador e duas professoras de natação infantil com aproximadamente dois anos de experiência. A sistematização das atividades teve como base a Pedagogia da Facilitação.

RESULTADOS: Foram relatadas as experiências do facilitador em cada etapa da Pedagogia da Facilitação. Na introdução, o facilitador expôs a proposta da comunidade de prática e junto com as professoras definiram a organização dos encontros. No questionamento, o facilitador indagou as professoras sobre os problemas enfrentados na atuação profissional e definiram uma estratégia para solucioná-los. Na experimentação, as professoras aplicaram a estratégia na prática. Na avaliação, o facilitador levantou questionamentos sobre as estratégias utilizadas e junto com as professoras avaliaram as experiências. No aprendizado, o facilitador propôs que as professoras refletissem sobre todos os encontros realizados e a influência sobre o novo conhecimento adquirido. O compartilhamento foi realizado informalmente após a aplicação da estratégia na prática.

CONCLUSÃo: Ao perfazer todas as etapas da Pedagogia da Facilitação, pode-se concluir que as professoras participantes da comunidade de prática conseguiram identificar os problemas que enfrentam na prática, definir estratégias para solucioná-los, experimentá-las e avaliar todo o processo na aprendizagem de novas estratégias. Desse modo, pode-se afirmar que a utilização da Pedagogia da Facilitação como uma proposta adequada a sistematização das atividades de uma comunidade de prática.

\section{ABSTRACT}

BACKGROUND: Communities of practice are groups of professionals who share a common interest. An essential element to conduct them is the facilitator. The latter has the function of organizing meetings, stimulating cooperation and assisting members in professional learning.

OBJECTIVE: report the experience of facilitating a community of practice for swimming teachers.

METHODS: a descriptive study was carried out in the form of an experience report by the facilitator of a community of practicing swimming teachers. Three teachers participated in this community, one who acted as facilitator and two teachers of infant swimming with approximately two years of experience. The systematization of activities was based on the Pedagogy of Facilitation.

RESULTS: The facilitator's experiences at each stage of the Pedagogy of Facilitation were reported. In the introduction, the facilitator exposed the proposal of the community of practice and together with the teachers defined the organization of the meetings. In questioning, the facilitator questioned the teachers about the problems faced in professional practice and defined a strategy to solve it. In experimentation, the teachers applied the strategy in practice. In the evaluation, the facilitator raised questions about the strategies used and together with the teachers evaluated the experiences. In learning, the facilitator proposed that the teachers reflect on all the meetings held and the influence on the new knowledge acquired. Sharing was carried out informally after the strategy was applied in practice.

CONCLUSION: By completing all the stages of the Pedagogy of Facilitation, it can be concluded that the teachers participating in the community of practice were able to identify the problems they face in practice, define strategies to solve them, experience them and evaluate the whole process in learning new ones. strategies. Thus, it can be said that the use of Pedagogy of Facilitation as an adequate proposal to systematize the activities of a community of practice. 


\section{INTRODUÇÃo}

O desenvolvimento profissional de professores de natação pode ocorrer em três contextos: formal, não formal e informal. O contexto formal é conhecido como os cursos de graduação e pós-graduação, nos quais o conhecimento a ser adquirido é predeterminado. O contexto não formal, são cursos de menor duração e mais específicos, incluindo oficinas, seminários, cursos de federações entre outros. Já o contexto informal são as mais variadas experiências de prática pessoal e profissional (NELSON; CUSHION; POTRAC, 2006; BRASIL et al., 2015).

Estudos sobre a formação e desenvolvimento profissional de professores e treinadores esportivos, têm evidenciado que, na percepção dos participantes investigados, o contexto informal tem maior influência ou significado no processo de aprendizagem. As diferentes situações deste contexto, permitem que o indivíduo construa conhecimentos, crenças e competências a partir das necessidades e desafios enfrentados no cotidiano de prática profissional (TSANGARIDOU, 2006; TRUDEL; GILBERT, 2006).

Dentro do contexto informal, as comunidades de prática (WENGER, 1998), têm atraído a atenção de pesquisadores, que buscam compreender como grupos de professores e treinadores aprendem ou se desenvolvem através do compartilhamento de informações e experiências. Para tanto, Ristow et al. (2020) identificou que professores se organizam em comunidades com diferentes propósitos: aprender a ensinar Educação Física, aprender a utilizar modelos de ensino, aprender a aplicar determinadas abordagens de ensino e desenvolver práticas de ensino.

As comunidades de prática são grupos de pessoas ou profissionais que compactuam de uma mesma paixão ou interesse. Que se relacionam frequentemente com o intuito de aprender sobre o domínio em comum (WENGER, 1998). A aprendizagem profissional ocorre pelo compartilhamento de experiências e conhecimentos, buscando soluções para os problemas enfrentados na prática. Os membros "trazem" para o grupo, toda a sua vivência, proporcionando aos colegas informações valiosas para possíveis resoluções de desafios da prática profissional (BRASIL et al., 2015).

Uma comunidade de prática é caracterizada por três dimensões: o empreendimento em conjunto, que é o tema ou assunto em comum. $O$ engajamento mútuo, referente a interação e compartilhamento de informações entre os membros. E o repertório compartilhado, que se refere aos "produtos" da comunidade, como: ações, estratégias, instrumentos e/ou práticas adotadas pelos membros (WENGER, 1998).

As comunidades de prática parecem ser um ambiente de aprendizagem colaborativa promissora no desenvolvimento profissional na área da Educação Física (CULVER; TRUDEL, 2008; GOODYEAR; CASEY; KIRK, 2014; YOON; ARMOUR, 2017; LUGUETTI et al., 2018). Um elemento considerado essencial na criação e desenvolvimento das comunidades é o facilitador. Sua função é realizar a gestão dos encontros, estimular a interação entre os membros, auxiliá-los no processo de reflexão e entre outras (CALLARY; WERTHNER; TRUDEL, 2013; HADAR; BRODY, 2020).

Para cumprir este papel com excelência, o facilitador deve ter conhecimento sobre as características dos participantes, competência no gerenciamento de dinâmicas, além de habilidades de comunicação, interpretação das falas e comprometimento com o grupo. Outro ponto a destacar é o posicionamento. O facilitador deve agir de modo que os membros se sintam no mesmo nível hierárquico, focando o seu discurso na cooperação entre os membros (HADAR; BRODY, 2020).

No entanto, parece existir uma lacuna na compreensão sobre a atuação do facilitador e estratégias desenvolvidas na gestão das comunidades de prática (RISTOW et al., 2020). Diante deste contexto, o objetivou-se relatar a experiência vivenciada na facilitação de uma comunidade de prática de professores de natação.

\section{MÉTODOS}

Este estudo se caracteriza como um estudo descritivo, na modalidade de relato de experiência, do facilitador de uma comunidade de prática de professores de natação. Fizeram parte desse grupo, um professor de natação (autor deste relato) com aproximadamente 10 anos de experiência, que atuou como facilitador. Além de trabalhar com natação infantil, o facilitador também é professor do curso de Educação Física do Centro Universitário de Brusque, Santa Catarina. A comunidade também foi composta por duas professoras com aproximadamente dois anos de experiência.

Este grupo de professores trabalha com natação infantil, com crianças entre nove e doze anos, em uma academia no município de Brusque, Santa Catarina. Este estabelecimento possui a estrutura de duas piscinas: uma com vinte e cinco metros de comprimento, composta por seis raias, onde são realizadas as aulas de crianças a partir dos oito anos de idade. A outra, de doze metros de comprimento com cinco raias, onde são realizadas as aulas de crianças a partir dos seis meses até os sete anos de idade.

A academia é credenciada pela Metodologia Gustavo Borges, na qual o nível pedagógico das crianças é determinado a partir da relação entre a idade cronológica e o nível de habilidade aquática. No contexto de atuação dos professores, as aulas das crianças entre nove e doze anos são realizadas na piscina de vinte e cinco metros e estão nos níveis denominados "aperfeiçoamento". Este nível é dividido em três (LIMA; BORGES, 2006).

O Aperfeiçoamento I é caracterizado como a "fase de aquisição de novos conceitos e habilidades aquáticas. Os nados crawl e costas são aperfeiçoados e o aprendizado do nado peito se inicia, exigindo dos alunos melhor execução técnica dos nados" (LIMA; BORGES, p. 50, 2006). O Aperfeiçoamento Il é o nível de "aperfeiçoamento das técnicas dos nados crawl, costas, peito e aprendizado do borboleta. Iniciação do condicionamento aeróbico e capacidade de nadar metragens mais longas" (LIMA; BORGES, p. 54, 2006). Já o Aperfeiçoamento III é a "Fase de aprimoramento técnico dos nados, em que as aulas são direcionadas para o condicionamento das variáveis fisiológicas e estímulos motivacionais. Preparação para ingressar numa equipe competitiva" (LIMA; BORGES, p. 58, 2006).

A origem desta comunidade aconteceu quando o facilitador, convidou os professores da academia para participarem de um grupo com o objetivo de desenvolver a prática pedagógica. Dos oito professores convidados, duas aceitaram em participar. Com o objetivo de organizar a comunidade e as atividades que 
seriam desenvolvidas, foi utilizada como base a Pedagogia da Facilitação (POEKERT, 2011), na qual é realizado um processo cíclico composto por seis etapas: introdução, questionamento, experimentação, avaliação, aprendizado e compartilhamento.

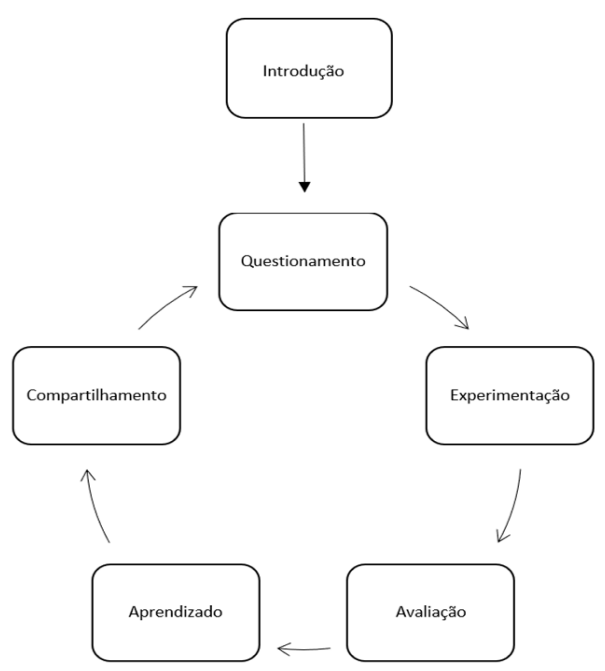

Figura 1. Etapas da pedagogia da facilitação. Fonte: elaborado pelo autor.

Após as experiências na facilitação de uma comunidade de prática, são descritas as ações do facilitador em cada uma destas etapas.

\section{RESULTADOS E DISCUSSÃO}

Na primeira etapa, denominada introdução, a função do facilitador é fazer uma explanação geral sobre a proposta da comunidade, esclarecendo dúvidas das professoras e pré-definindo o calendário dos encontros (POEKERT, 2011). Assim, o facilitador fez uma breve explanação sobre o conceito de comunidades de prática diferenciando-as de outros grupos de profissionais como networking, grupo de estudos etc. Para Wenger, McDermott e Snyder (2002) comunidades de prática são grupos de pessoas que compartilham um conjunto de problemas sobre um tópico ou tema bem definido e que juntos, interagem de forma permanente para desenvolver suas competências.

Ainda nesta etapa, o facilitador solicitou que fosse definida a frequência, dia da semana, horário e duração dos encontros. Em comum acordo, os três membros definiram que os encontros seriam realizados pelo aplicativo Google Meet, quinzenalmente nas terças-feiras às $13 \mathrm{~h} 45$, com a duração de no máximo uma hora. Além disso, como tarefa para o encontro seguinte e dando início a segunda etapa, questionamento, o facilitador pediu para as professoras refletirem sobre as dificuldades, desafios ou problemas que elas se deparam ao ministrar aulas de natação infantil.

A etapa de questionamento dá início ao processo de desenvolvimento profissional propriamente dito. Para isso, é preciso que os membros da comunidade identifiquem os problemas que desejam enfrentar em conjunto, definindo assim, o empreendimento em conjunto. Nesta etapa, o facilitador deve estimular os membros a identificarem as suas necessidades por meio de questionamentos (POEKERT, 2011). Para isso, o facilitador pediu para que as professoras, escrevessem uma lista com o auxílio da ferramenta Jamboard. Após fazer o levantamento dos desafios, o facilitador pediu para que as professoras realizassem uma explicação verbal, aprofundando no tema.

Nesta etapa é essencial que os membros da comunidade sintam que eles são responsáveis pelo desenvolvimento profissional e assumam a responsabilidade na resolução dos problemas uns dos outros. Esta iniciativa é fundamental na aprendizagem colaborativa. Deste modo, o facilitador deve promover o empoderamento dos membros, posicionando-se como um auxiliar ou um colaborador do processo (TANNEHILL; MACPHAIL, 2017).

As professoras, através das indagações do facilitador, definiram que o principal problema a ser enfrentado em suas aulas, era o comportamento dos alunos e que este problema teria origem no método de ensino diretivo e tecnicista que elas utilizavam. Ainda na etapa de identificação, o facilitador deve instigar os membros a pensarem sobre possíveis estratégias para solucionar o problema (POEKERT, 2011). Após discutirem sobre algumas possibilidades, o facilitador sugeriu que no próximo encontro fosse debatido a respeito dos modelos de instrução, com base na resenha do livro de Metzler (2011) elaborada por Rufino e De Souza Neto (2016). As professoras aceitaram a proposta e o facilitador se voluntariou para enviar o artigo aos demais membros e fazer uma breve explanação na introdução do encontro seguinte.

Uma das funções do facilitador é de instigar pensamentos e reflexões, ao invés de transmitir conhecimentos (CALLARY; WERTHNER; TRUDEL, 2013). Neste momento, ao propor algumas estratégias ou possibilidades, parece que o facilitador, se posicionou mais como um membro da comunidade do que um facilitador propriamente dito. De fato, o facilitador é considerado um membro. $O$ equilíbrio entre a facilitação e a participação como membro é um ponto extremamente sensível, em que o indivíduo que exerce a facilitação deve estar constantemente analisando.

Ainda na etapa de questionamento, no encontro seguinte, após a explanação, o facilitador questionou as professoras sobre qual modelos elas acreditavam ser o mais adequado para ser usado como solução dos problemas levantados. Para elas, tanto o modelo Peer teaching quanto o Collaborative learning pareceram ser convenientes, pois ambos tornam os alunos responsáveis pelo processo de ensino e aprendizagem dos colegas. Então, o facilitador sugeriu que o grupo discutisse sobre como seria uma aula de natação utilizando os modelos selecionados, com o objetivo de escolher apenas um.

Durante a discussão, surgiram várias dúvidas sobre a aplicação dos modelos. Após análise dos modelos, a escolha foi pelo Peer teaching. Já que para as professoras, este modelo se assemelha com o método já utilizado por elas e não precisariam fazer muitas modificações no planejamento e na intervenção. Antes de passarem para a próxima etapa, experimentação, o facilitador sugeriu que as professoras realizassem um plano de aula para aplicar na semana seguinte. E assim, no próximo encontro, fosse realizada a etapa de avaliação.

Dessa forma, as professoras foram orientadas para que, ao final das aulas ministradas, fosse enviada uma mensagem de áudio pelo aplicativo WhatsApp, descrevendo a aula e apresentado suas reflexões sobre os pontos positivos e negativos da 
experiência. Após o envio das mensagens, o facilitador analisou as falas e retornou as mensagens com frases encorajadoras e questionamento sobre como poderia ser aperfeiçoado a aplicação deste modelo no ensino da natação. De fato, nesta etapa, a facilitação deve assumir uma postura relacionando motivação e questionamento, para que o processo de desenvolvimento seja "apoiado" pelo facilitador.

No encontro realizado após as experiências práticas, foi realizada a avaliação. Nesta etapa, o facilitador deve escutar o relato dos membros e instigá-los a refletir sobre os resultados alcançados da escolha da solução para a resolução do problema (POEKERT, 2011). Dessa forma, o facilitador solicitou que cada professora descrevesse as suas aulas ministradas com o modelo Peer teaching. Após a descrição detalhada das aulas, o facilitador iniciou uma reflexão analisando se o problema foi solucionado e os motivos que levaram a essa resolução.

Para as professoras, posicionar os alunos como tutores de seus colegas, fez com que eles demonstrassem mais interesse na aula. Eles participaram ativamente da aula, analisando o nado dos colegas, realizando correções, dando sugestões e dicas. Embora o foco das aulas ainda fosse o desenvolvimento técnico, a mudança na participação dos alunos, de reprodutores de movimentos técnicos para produtores, parece ter sido o principal motivo da resolução do problema.

Na etapa seguinte, aprendizado, o facilitador deve estimular os membros a analisarem todo o processo e refletirem sobre os sucessos e fracassos na aprendizagem de novas estratégias, métodos ou modelos (POEKERT, 2001). Dessa forma, ainda no mesmo encontro o facilitador pediu para que as professoras recordassem todos os encontros e apontassem os momentos mais importantes e os que não tiveram influência no aprendizado. No contexto relatado, a etapa de compartilhamento foi realizada informalmente após a intervenção das professoras, tanto pelas mensagens no aplicativo WhatApp, quanto no último encontro.

O desenvolvimento ou a análise dos resultados da participação de uma comunidade de prática pode ser avaliada a partir do framework de criação de valores proposto por Wenger, Trayner e De Laat (2011). Neste são determinados indicadores que permitem ao facilitador analisar se as atividades propostas, a interação ou se os objetivos foram bem-sucedidos. O framework é composto por cinco valores, que juntos compõem a criação de valor: i) Valor imediato, quando o participantes logo nas primeiras reuniões acreditam que a sua participação no comunidade pode gerar frutos; ii) Valor potencial, quando é desenvolvido algum tipo de conhecimento; iii) Valor aplicado, quando o Valor potencial gera mudanças na prática; iv) Valor realizado, quando as mudanças na prática resultam na melhora no desempenho; e v) Valor reformulado, quando todo o processo é avaliado e a criação de valor é alcançada.

Acredita-se que no contexto do presente relato de experiência, as professoras identificaram que ter participado de uma comunidade foi valioso. A partir do framework de Wenger, Trayner e De Laat (2011), percebe-se que elas, desde o primeiro encontro acreditaram que seria produtivo participar dos encontros (Valor imediato). Adquiriram conhecimentos sobre um modelo de instrução (Valor potencial). Aplicaram este conhecimento em suas aulas (Valor aplicado). As professoras perceberam que o problema identificado no segundo encontro foi solucionado (Valor realizado). Por fim, ao refletirem sobre todo o processo, elas avaliam ter sido influente no desenvolvimento profissional (Valor reformulado).

\section{CONCLUSÃO}

O objetivo pessoal em escrever um relato de experiência de facilitação, foi apresentar uma maneira de estruturar sistematicamente os encontros de uma comunidade de prática. Como foi a primeira vez que havia assumido o papel de facilitador, surgiram alguns sentimentos, como: motivação, entusiasmo, medo, insegurança, entre outros. Os sentimentos positivos, estavam relacionados a participação em algo novo e que poderia resultar no meu desenvolvimento profissional e de meus colegas. Já os sentimentos negativos, estavam relacionados a pressão sobre a minha atuação enquanto facilitador, uma vez que a participação das professoras era voluntaria e os encontros deveriam fazer sentido para elas.

Dessa forma, ao investigar na produção cientifica sobre facilitação e organização de comunidades de prática, pude perceber que a sistematização das atividades conforme propõe Poekert (2011) seria uma escolha adequada. De fato, foi, no início, acredito que permitir o envolvimento ativo das participantes na definição do tema ou do conteúdo, foi essencial para gerar o engajamentos delas e contribuiu para que elas dessem significados às atividades desenvolvidas nos encontros. Além disso, acredito que a frequência e duração dos encontros foram adequados para manter o equilíbrio entre a motivação e a agenda de compromissos dos participantes.

Por fim, acredita-se que este relato possa contribuir com a criação, manutenção e organização de futuras comunidades. Acredita-se que o bom relacionamento entre facilitador e membros é um ponto fundamental para gerar confiança no processo de aprendizagem. Dessa forma, se sugere que antes de iniciar as atividades de aprendizagem, o facilitador conheça bem os participantes e vice-versa. Além disso, a sua fala deve sempre ser sincera e transparente.

\section{REFERÊNCIAS}

BRASIL, V. Z.; RAMOS, V.; DA SILVA DE BARROS, T. E.; GODTSFRIEDT, J.; VIEIRA DO NASCIMENTO, J. A trajetória de vida do treinador esportivo: as situações de aprendizagem em contexto informal. Movimento, Porto Alegre, v. 21, n. 3, p. 815-29, 2015

CALLARY, B.; WERTHNER, P.; TRUDEL, P. Exploring coaching actions based on developed values: A case study of a female hockey coach. International Journal of Lifelong Education, Nottingham, v. 32, n. 2, p. 209-29, 2013.

CULVER, D.; TRUDEL, P. Clarifying the concept of communities of practice in sport. International Journal of Sports Science \& Coaching, Teller Road, v. 3 n. 1, p. 1-10, 2008.

GOODYEAR, V. A.; CASEY, A.; KIRK, D. Tweet me, message me, like me: Using social media to facilitate pedagogical change within an emerging community of practice. Sport, Education and Society, London, v. 19, n. 7, p. 927-43, 2014.

HADAR, L. L.; BRODY, D. L. Interrogating the role of facilitators in promoting learning in teacher educators' professional communities. Professional Development in Education, Leeds, v. 46, n. 1, p. 1-14, 2020.

LIMA, W. U.; BORGES, G. Manual formativo da metodologia Gustavo Borges. 3. ed. São Paulo: BPR, 2006.

LUGUETTI, C. ARANDA, R · ENRIQUEZ, O. N · OLIVER, K. L. Developing teachers' pedagogical identities through a community of practice: Learning to sustain the use of a student-centered inquiry as curriculum approach. Sport, Education and Society, London, v. 24, n. 8, p. 1-12, 2018.

METZLER, M. W. Instructional models for physical education. 3. ed. Scotts- 
dale: Holcomb Hathaway, 2011.

NELSON, L.; CUSHION, C.; POTRAC, P. Formal, non formal and informal coach learning: a holistic conceptualization. International Journal of Sports Science and Coaching, Leeds, v. 1, n. 3, p. 247-59, 2006.

POEKERT, P. The pedagogy of facilitation: Teacher inquiry as professional development in a Florida elementary school. Professional Development in Education, Leeds, v. 37, n. 1, p. 19-38, 2011.

RISTOW, L.; BACKES, A. F.; BIANCHESSI, D.; BRASIL, V. Z.; DA ROSA, R. S.; CARDOSO, J.; RAMOS, V. Comunidade de prática na formação e desenvolvimento profissional na educação física: uma metassíntese. Caderno de Educação Física e Esporte, Marechal Cândido Rondon, v. 18, n. 3, p. 117-23, 2020.

RUFINO, L. G. B.; DE SOUZA NETO, S. Novos modelos curriculares para a Educação Física brasileira: resenha do livro "Instructional Models for Physical Education - 3. ed". Motrivivência, Florianópolis, v. 28, n. 47, p. 290-304, 2016.

TANNEHILL, D.; MAcPHAIL, A. Teacher empowerment through engagement in a learning community in Ireland: Working across disadvantaged schools. Professional Development in Education, Leeds, v. 43, n. 3, p. 334-52, 2017.
TRUDEL, P.; GILBERT, W. Coaching and coach education. In: KIRK, D.; McDONALD, D.; O'SULLIVAN, M. (Ed.). Handbook of Physical Education. London: Sage, 2006. p. 516-39.

TSANGARIDOU, N. Teachers' beliefs. In: KIRK, D.; McDONALD, D.; O'SULLIVAN, M. (Ed.). Handbook of Physical Education. London: SAGE, 2006. p. 486-501.

WENGER, E. Communities of practice: learning, meaning and identity. Cambridge: Cambridge University Press, 1998

WENGER, E.; McDERMOTT, R. A.; SNYDER, W. Cultivating communities of practice: A guide to managing knowledge. Harvard Business Press, 2002.

WENGER, E.; TRAYNER, B.; DE LAAT, M. Promoting and assessing value creation in communities and networks: A conceptual framework. The Netherlands: Ruud de Moor Centrum, Heerlen, v. 20, p. 2010-11, 2011.

YOON, K.; ARMOUR, K. M. Mapping physical education teachers' professional learning and impacts on pupil learning in a community of practice in South Korea. Physical Education and Sport Pedagogy, London, v. 22, n. 4, p. 427-44, 2017.

\section{AGRADECIMENTOS}

Agradeço a Larissa e a Jennifer por confiarem na proposta e no desenvolvimento desta comunidade de prática.

\section{CONFLITO DE INTERESSE}

O autor do estudo declara não haver conflito de interesses

\section{FINANCIAMENTO}

Este estudo não teve apoio financeiro.

\section{ORCID E E-MAIL DO AUTOR}

Leonardo Ristow (Autor Correspondente)

ORCID: 0000-0002-2829-7737.

E-mail: leonardoristow@live.com 